\title{
Efficient Face Mask Recognition System by Using Deep Learning Methodology
}

\author{
Hari Priya $\mathrm{K}^{\mathrm{a}, 1}$ and Malathi $\mathrm{S}^{\mathrm{b}}$ \\ ${ }^{a}$ PG Scholar, Dept of CSE, Panimalar Engineering College, Chennai, India \\ ${ }^{b}$ Professor, Dept of CSE, Panimalar Engineering College, Chennai, India
}

\begin{abstract}
In this project, mask Recognition System is presented, that utilizes the prospect of Object Detection, completed the assistance of Deep Learning philosophies. The past work gives the topic of article identification by joining coarse-grained and fine-grained discovery philosophies without precedent for police work the moving items on high goal recordings. Period object location and acknowledgment finds careful applications in various fields like clinical applications, security police examination, and independent vehicles. There unit of estimation a few machine and profound learning procedures that unit utilized for object discovery and acknowledgment. The development of a convolutional neural organization $(\mathrm{CNN})$ has given a major forward leap to protest discovery and acknowledgment. Convolutional Neural Network (CNN) has arrived at the exemplification of picture characterization for different application. Explicitly in 2D-CNN, there's huge advancement for object discovery, beside 3D-CNN, it's still toward the start of partner time. Profound CNN's unit acclimated get extra exact directions and to deal with high goal video outlines. By taking this idea of location on the grounds that the base toour work, the framework includes in perceiving the essence of the individual and checks if the face have mask or not, befittingly guaranteeing the individual complies with the assurance safety measures so as that the unfurl of hepatotoxic infection may be managed. The framework will unpredictably be utilized for future face ID with face mask till the pandemic gets died.
\end{abstract}

Keywords: Face Mask, Recognition, CNN, Deep Learning, face ID.

\section{Introduction}

Deep learning is Associate in Nursing AI work that mirrors the functions of the human mind in procedure information to be used in examination objects, perceiving discourse, deciphering dialects, and making options. Deep learning AI is all through a hold to travel looking out while not human bearing, drawing from information that is each unstructured and unlabelled. Deep Learning could likewise be an AI procedure upheld neural organization structures with various layers of strategy units, that has been effectively applied to an expansive arrangement of issues among the territories of picture acknowledgment and correspondence method.

${ }^{1}$ Hari Priya K, PG Scholar, Dept of CSE, Panimalar Engineering College, Chennai, India. Email:priyahari827@gmail.com ${ }^{1}$ 
Facial acknowledgment includes abuse figuring to distinguish human countenances in pictures or recordings, along these lines mensuration explicit facial qualities. This may typify the hole among eyes, thus the general places of the nose, jaw and mouth. This data is consolidated to make a facial mark, or profile. Pundits of veil acknowledgment conjointly expect that this new innovation might be inclined to assortment of the indistinguishable entanglements as face acknowledgment. Afew of the instructing datasets utilized for biometric distinguishing proof are overwhelmed by caucasian individuals. In the AI model, Convolutional Neural Network are created abuse Python, the $\mathrm{CNN}$ model is that the best fitted to picture data grouping. When the picture has been preprocessed by abuse AN adequate instrument, it will be served to the fluctuated layers of the DCNN model. At stretches the $\mathrm{CNN}$ model, there are quantities of covered up layers that is made of neurons.

\section{Related work}

Sammy V. Militante; Nanette V. Dionisio, "Real-Time Facemask Recognition with AlarmSystem using Deep Learning", 2020 Behind the scenes of the COVID-19 pandemic, organizations, for example, the foundation experience the ill effects of basically shut all around the world if the current circumstance won't change. Corona virus otherwise called Serious Acute Respiratory Syndrome Corona infection 2 is an irresistible sickness that is delivered from a tainted debilitated individual who talks, wheezes, or hacks by respiratory drops. The dataset gathered contains 25,000 pictures utilizing 224x224 pixel goal and accomplished an exactness pace of $96 \%$ with regards to the presentation of the prepared model. The framework builds up a Raspberry Pibased constant facemask acknowledgment that cautions and catches the facial picture if the individual distinguished isn't wearing a facemask. This investigation is gainful in battling the spread of the infection and evading contact with the infection.

Hariri Walid, "Efficient Masked Face Recognition Method during the COVID19Pandemic", 2020 The COVID-19 is an unmatched emergency prompting countless setbacks and security issues. To diminish the spread of Covid, individuals regularly wear covers to secure themselves. This makes face acknowledgment a troublesome undertaking since specific pieces of the face are covered up. At last, the Bag-ofhighlights worldview is applied on the element guides of the last convolutional layer to quantize them and to get a slight portrayal contrasting with the completely associated layer oftraditional CNN. At long last, Multilayer Perceptron (MLP) is applied for the arrangement cycle. Trial results on Real-World-Masked-Face-Dataset show high acknowledgment execution.

Dan Zeng, Raymond Veldhuis and Luuk Spreeuwers," A survey of face recognition techniques under occlusion", 2020 To start with, we investigate what the impediment issue is and what inalienable troubles can emerge. As a piece of this audit, we present face identification under impediment, a primer advance in face acknowledgment. Second, we present how existing face acknowledgment strategies adapt to the impediment issue and characterize them into three classifications, which are 1) impediment powerful component extraction approaches, 2) impediment mindful face acknowledgment draws near, also, 3) impediment recuperation based face acknowledgment draws near. Moreover, they break down the inspirations, advancements, experts and cons, and the presentation of agent approaches for correlation. 
Walid Hariri, "Efficient Masked Face Recognition Method During Thecovid-19 Pandemic", 2020 The COVID-19 is an unrivaled emergency prompting an enormous number of losses and security issues. To lessen the spread of Covid, individuals frequently wear veils to secure themselves. This makes face acknowledgment a troublesome assignment since specific pieces of the face are covered up. At long last, the Bag-of-highlights worldview is applied on the include guides of the last convolutional layer to quantize them and to get a slight portrayal contrasting with the completely associated layer of old style CNN. At last, Multilayer Perceptron (MLP) is applied for the grouping cycle. Test results on Real-World-Masked-Face-Dataset show high acknowledgment execution.

Renliang Weng, Singapore; Jiwen Lu; Yap-Peng Tan," Robust Point Set Matching for Partial Face Recognition", 2018 In the course of recent many years, various face acknowledgment techniques have been proposed in PC vision, and the majority of them utilize comprehensive face pictures for individual distinguishing proof. At that point, propose a hearty direct set coordinating with strategy toward discriminatively match these two extricated neighborhood include sets, where both the textural data and mathematical data of nearby highlights are unequivocally utilized for coordinating at the same time. At last, the likeness of two appearances is changed over as the distance between these two adjusted capabilities. Trial results on four public face informational indexes show the adequacy of the proposed approach.

\section{Architecture}

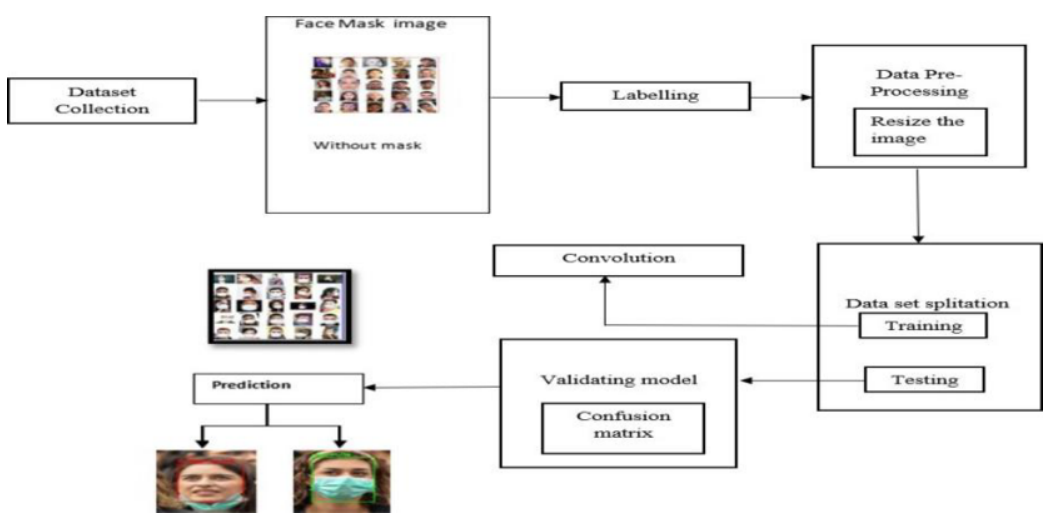

Figure 1. Face Mask recognition system architecture

Data sets are collected together. After Collecting images together, labeling the image and enter into training and test set. Collected images will test and train using deep learning with CNN. After finishing training set and test set the model will be saved. Then running the webcam which loaded the image model for live detection. Finally the face will be detect whether the face in image carry face mask or not. Predict the image whether it carry face mask or not. 


\section{Results and Discussion}

Data assortment is that the initial step within the method of evaluating the outcomes and therefore the collected dataset is pre-processed to get rid of any noise or outliers if gift. Datasets area unit associate degree integral a part of machine learning. The main advantage of this field may result from advances in machine learning algorithms, constituent, and, less-intuitively, the provision of high-quality coaching datasets. The knowledge labeling, within the text of machine learning, is that the method of detective work and tagging knowledge samples. The method are often manual however is sometimes performed by the part of code as Figure 2 .
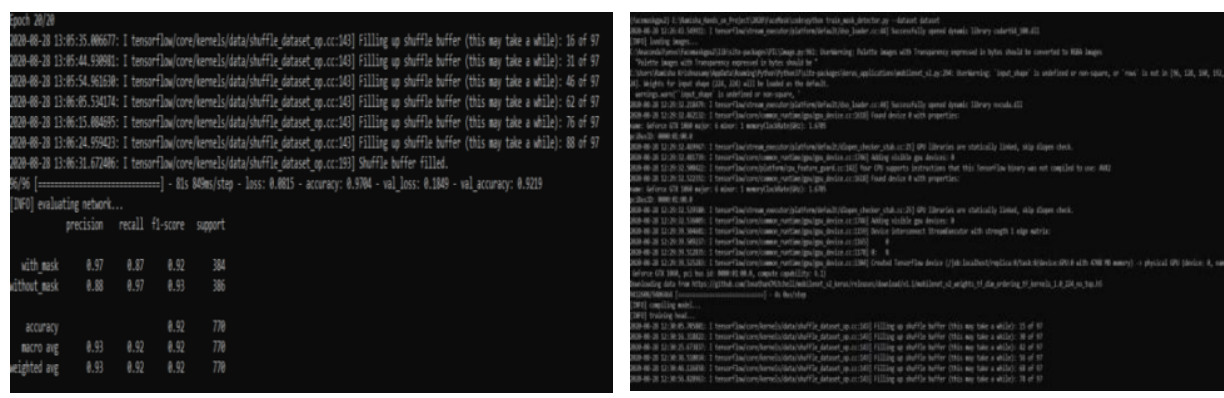

Figure 2. Execution result

\section{Performance Metrices}

The significance execution grid is a way to deal with focusing on which parts of execution ought to bedependent upon progress. In this system we get $92 \%$ of accuracy by test the dataset. Let us see the performance of proposed system in table 1:

Table 1. Performance analysis

\begin{tabular}{lllll}
\hline $\begin{array}{l}\text { No of input } \\
\text { image(training) }\end{array}$ & $\begin{array}{l}\text { No of input } \\
\text { image (test) }\end{array}$ & Epoch & Training set accuracy & $\begin{array}{l}\text { Test set } \\
\text { accuracy }\end{array}$ \\
\hline 50 & 10 & 20 & 75 & 45 \\
100 & 20 & 20 & 85 & 45 \\
500 & 50 & 20 & 86 & 60 \\
1000 & 200 & 20 & 89 & 76 \\
1500 & 300 & 20 & 90 & 89 \\
1900 & 400 & 20 & 92 & 92 \\
\hline
\end{tabular}

\section{Conclusion}

Datasets area unit associate degree integral a part of the sphere of machine learning. Major advances during this field may result from advances in learning algorithms (such as deep learning), constituent, and less-intuitively, the provision of high-quality training datasets. In this section, Training and therefore the Testing datasets area unit loaded that consecutive step during this method. The photographs and captions needed 
for the model area unit subjected to pre- processing and therefore the developed models area unit incorporate along to produce the expected framework. Finally the model is saved, and therefore the prediction begins.

\section{Future goal}

The objective of face recognition is, from the incoming image, to find a series of data of the same face in a set of training images in a database. The great difficulty is ensuring that this process is carried out in real-time, something that is not available to all biometric facial recognition software providers. Future works incorporate the combination of physical separating, wherein the camera distinguishes the individual wearing a facemask or not and simultaneously gauges the distance between every individual and makes an alert if the physical removing doesn't notice as expected.

\section{References}

[1] Sammy V. Militante; Nanette V. Dionisio, Real-Time Facemask Recognition with Alarm System using DeepLearning, 2020.

[2] Hariri Walid, Efficient Masked Face Recognition Method during the COVID-19 Pandemic, 2020.

[3] Dan Zeng, Raymond Veldhuis and Luuk Spreeuwers, A survey of face recognition techniques underocclusion, 2020.

[4] Walid Hariri,Efficient Masked Face Recognition Method During TheCovid-19 Pandemic, 2020.

[5] Renliang Weng, Singapore; Jiwen Lu; Yap-Peng Tan, Robust Point Set Matching for Partial FaceRecognition, 2018.

[6] Deqiang He, Zikai Yao, Zhou Jiang, Yanjun Chen, JiDeng and Weibin xiang, Detection of Foreign Matter on High-Speed Train Underbody Based on Deep Learning.

[7] Dong-ju Jeong, Insung Hwang, and Nam Ik Cho, Senior Member, IEEE., Co-salient Object Detection Based on Deep Saliency Networks and Seed Propagation over an Integrated Graph.

[8] Dong Kyun Shin, Minhaz Uddin Ahmed and PhillKyu Rhee, Incremental Deep Learning for Robust Object Detection in Unknown Cluttered Environments.

[9] Faisal Mehmood, Israr Ullah, Shabir Ahmad, DoHyeun Kim.,Object detection mechanism based on deep learning algorithm using embedded IoT devices for smart home appliances control in CoT Journal of Ambient Intelligence and Humanized Computing.

[10] Florin C. Ghesu, Bogdan Georgescu, Member, IEEE, Yefeng Zheng, Senior Member, IEEE, SasaGrbic, Andreas Maier, Joachim Hornegger, and Dorin Comaniciu, Fellow, IEEE, Multi-Scale Deep Reinforcement Learning for Real-Time 3D-Landmark Detection in CT Scans.

[11] Ambeth Kumar.V.D, Dr.M.Ramakrishnan, V.D.Ashok Kumar and Dr.S.Malathi (2015).Performance Improvement using an Automation System for Recognition of Multiple Parametric Features based on Human Footprint. kuwait journal of science, Vol 42, No 1 (2015), pp:109-132.

[12] Ambeth Kumar.V.D, V.D.Ashok Kumar, S.Malathi, K.Vengatesan, M.Ramakrishnan.Facial Recognition System For Suspect Identification Using A Surveillance Camera.Pattern Recognition and Image Analysis (Springer), Volume 28, Issue 3, pp 410-420, 2018. 10.1134/S1054661818030136)

[13] Ambeth Kumar.V.D and M.Ramakrishan. Employment Of Footprint Recognition System. in the month of December for Indian Journal of Computer Science and Engineering (IJCSE) Vol. 3 No.6 Dec 2013

[14] Ramya,T.,Dr.Malathi,S.,ratheeksha,G.R. and Dr.V.D.Ambeth Kumar (2014).Personalized authentication procedure for restricted web service access in mobile phones.Applications of Digital Information and Web Technologies (ICADIWT), 2014, Page(s):69 - 74, Bangalore, India (ISBN:978-14799-2258-1)

[15] V.D.Ambeth Kumar (2018). Novel Wireless Sensing System For The Welfare Of Sewer Laborers. Healthcare Technology Letters (IET) Volume 5, Issue 4, p. $107-112$.

[16] V.D.Ambeth Kumar et.al, .IOT Based Smart Museum Using Wearable Device. Lecture Notes in Networks and Systems, Vol.55, pp: 33-42, 2018 